\title{
CLINICAL OUTCOMES OF COMBINED SIMULTANEOUS FEMTOSECOND KERARINGS IMPLANTATION AND CORNEAL COLLAGEN CROSS-LINKING IN ADVANCED KERATOCONUS
}

\author{
Abdelwhad, N. ${ }^{1(*)}$, Mohamed, M. ${ }^{1}$, Yousef, H. ${ }^{1} \&$ Mounir, A. ${ }^{2}$ \\ ${ }^{1}$ Ophthalmology dept., Faculty of Medicine, AL-Azhar Univ., Assuit, Egypt \\ ${ }^{2}$ Ophthalmology dept., Faculty of Medicine, Sohag Univ., Sohag, Egypt \\ *E-mail: neven.matwdaa@gmail.com
}

\begin{abstract}
Aim of Work: To study the clinical outcomes of combined simultaneous femtosecond kerarings implantation and corneal collagen cross-linking in advanced keratoconus. Methods: A prospective study was conducted on 25 eyes of 33 patients were diagnosed as advanced keratoconus. The study population underwent combined simultaneous femtosecond kerarings implantation and corneal collagen cross-linking. Results were reported at the $1^{\text {st }}, 3^{\text {rd }}, 6^{\text {th }}$ and $12^{\text {th }}$ months post-operatively Results: a significant improvement was found in postoperative (UCVA) (BCVA) and (SER) started form the first month postoperatively and continued all over the year $(P<0,001)$. The flattest $k$ (K1) the steepest $k$ (K2) and the average $k$ (Ka) showed marked decrease after 3 month and became just stable in next 3 months and increase slightly thereafter $(P=0.001)$. Conclusions: Combined simultaneous femtosecond kerarings implantation and corneal collagen cross-linking is an effective method in treating cases with advanced keratoconus
\end{abstract}

Keywords: Advanced keratoconus femtosecond laser, Keraring, Cross linking

\section{Introduction}

Keratoconus (KC) is now regarded as a treatable less rare disease with increasing incidence and a rising prevalence that approaches 3\% amongst adolescents and young adults in certain geographic areas [1]. It is a disorder in which the cornea supposes a conical form as a consequence of non-inflammatory advanced corneal thinning. The thinning and protrusions in keratoconus persuades asymmetrical astigmatism with or with no myopia, causing mild to noticeable damage in vision quality and amount [2]. Glasses and contact lenses are the common treatments in the primary grades of keratoconus [3]. Intrastromal corneal rings (intacs), that was made initially for the corrections of mild to moderate myopia, are nowadays being studied for keratoconus corrections with clear corneas [4]. The objective of ICRS ("kerarings" \& ferrara) implantations is to advance visual acuity with no removing any corneal tissues or touch the central cornea. In progressive keratoconus with corneal opacities, corneal grafting may be the only operative treatment substitute. A novel method of collagen 
cross-linking by the photo-sensitizer riboflavin and UVA identical to photopolimerization was settled. Concentrated experimental reports revealed a significant rise in corneal rigidity by about 70 percent in non-treated vs. treated corneas thereafter collagen cross-linking [5]. Thus stiffening the cornea with collagen cross linking

\section{Patient and Methods}

The design of this study was (prospective non-randomized) clinical report was conducted in future femto-lasik \& cornea center Sohag, Egypt 25 eyes of 33 patients was diagnosed as having advanced keratoconus were eligible for this study. Inclusion criteria were as follows: age between 18-35 year, patients with clear cornea with advanced keratoconous, keratometry average $60>\mathrm{ka}>55 \mathrm{ds}$, thinnest location less than 380 um and more than 330 um and depth at implantation site equal or more than 450 at $5 \mathrm{~mm}$ from the midpoint of the cornea. Patient with corneal opacity, hydrops or scarning, ocular traumatic history, ocular surgeries and other corneal diseases, manifestation of severe dry eye, and history of systemic diseases that may affect the cornea as autoimmune diseases and diabetes were excluded. All

\subsection{Surgical technique}

Preparation of the operation included prophylactic topical anti-biotic eyes droplets e.g. Gatifloxacin $0.30 \% 5$ doses daily 24 hours before surgery, then topical Anesthesia e.g: Benoxinate hydro-chloride $0.40 \%$ had been used 1 hour before operation followed by topical antisepsis Povidone iodine. The parameters of Femto-second laser for the corneal tunnel were: internal diameter: $5 \mathrm{~mm}$, external diameter: $5.9 \mathrm{~cm}$, depth: $80.0 \%$ of thinnest central corneal thickness, incision location at the axis of K2 (the steepest) corneal meridian. Energy $2.00 \mathrm{Mj}$, after ring implantation, transepithelial corneal collagen cross linking, which

\subsection{Follow up}

Patients were examined in 1 month, 3 months, 6 months, 12 months, all patents were examined for a complete ophthalmological tests include UCVA BCVA refraction may discontinue the advance of the disorder and turn it to form fruste [6]. The current work aimed to evaluate the clinical outcomes of combined simultaneous femtosecond kerarings implantations and corneal collagen crosslinking in advanced keratoconus .

kertoconic eyes of the study had been subjected to combined corneal collagen cross linking and implantation corneal ring segment (keraing) (Mediphacos, Belo Horizonte, Brazil). The devices used in this study were: The KXL® System (Avedro, Waltham, Massachusetts, USA). and Advanced Femtosecond Laser (iFS; Abbott, Chicago, Illinois, USA). Full Ophthalmic examination had been performed on all eyes before surgery included Un Corrected Visual acuty (UCVA) in log MAR, Best Corrected Visual acuity (BCVA) in $\log$ MAR, corneal topography, corneal aberrometry and mesopic pupil diameter were assessed by means of Sirius Scheimpflug-placido topography (CSO, Italy), slit lamp examination and fundus examination.

include dropping of riboflavin. (Para Cel, Avedro, Waltham, Massachusetts, USA) on the cornea every $1.5 \mathrm{~min}$ for $4.5 \mathrm{~min}$. Then dropping of riboflavin (Vibex Xtra) every one and half minutes for 6 minutes. Trailed by 5.2 -min accelerated CXL by means of the pulsing style with $45 \mathrm{~mW} /$ $\mathrm{CC}$ power. The post-operative drugs comprised topical anti-biotics eyes drops (e.g. Gatifloxacin $0.30 \% 5$ times daily for 7 days), Topical steroid eyes drops (e.g. Prednisolone acetate $1 \% 5$ times daily for 7 days), Lubricant eyes drops 5 times daily, and systemic nonsteroidal antiinflammatory medications.

spherical equivalent $\mathrm{k} 1$, $\mathrm{k} 2$ average $\mathrm{k}$, pachymetry and scheimflug imaging with pentacam (sirus) device. 


\subsection{Statistical analysis}

Analysis of the collected data has been performed via STATA v-14.2 (Stata Statistical Package, College Station, TX: StataCorp LP.). Quantitative data has been introduced as means, $\mathrm{SD}$, median and range. Comparisons were performed amid preoperative and post-operative followingup data at 12,36 -mths and 60-mths by

\section{Results}

A total of 25 eyes of 33 patents are subjected to simultaneous combination of accelerated corneal collagen cross linking and femtolasik keraring implantation with follow up for a period of one year. In our study the un UCVA exhibited progress from $1.1 \pm 0.34$ to $0.72 \pm 0.15$ ( $\mathrm{P}<$ 0.0001), tab. (1) \& fig. (1) The BCVA exhibited progress from $0.84 \pm 0.09$ to $0.52 \pm 0.14$ at $12^{\text {th }}$ month $(\mathrm{P}<0.0001)$, tab. (2) \& fig. (2). Spherical equivalent refraction showed improvement from -7.34 \pm 4.29 to $-4.20 \pm 3.01$ at $12^{\text {th }}$ Month, $(\mathrm{P}$ $<0.0001)$ The Flattest K, steepest K and means of RMANOVA testing. Sphericity were tested by means of Mauchly's Testing of Sphericity. Bonferroni post-hoc testing has been utilized to study the differences at each time point. Graphs have been made via Microsoft Excel. Significance was considered at $\mathrm{P}<0.05$.

average $\mathrm{K}$ showed marked decrease the flattest $\mathrm{K}$ decreased from $51.50 \pm 5.16$ to $50.49 \pm 5.42(\mathrm{P}=0,002)$. The steepest $\mathrm{K}$ decreased from $56.98 \pm 6.20$ to $53.47 \pm$ 5.62 and the average $\mathrm{K}$ decreased from $53.87 \pm 5.42$ to $51.88 \pm 5.40$. $(\mathrm{P}=0.001)$, tab. (3) \& fig. (3). The flattening affect with keraring was remarkable in pentacam kerometry maps and regularization of corneal surface was also noticed by scheimflug imaging after keraring implantation and cross linking all over the period of follow up, fig. (4).

Table 1: UCVA over time preoperative and postoperative

\begin{tabular}{|c|c|c|}
\hline Time & UCVA & $P$ value for repeated measures \\
\hline Preoperative: Mean \pm SD & $1.1 \pm 0.34$ & \multirow{5}{*}{$<0.0001$} \\
\hline Postoperative 1 month: Mean \pm SD & $1.02 \pm 0.21$ & \\
\hline Postoperative 3 month: Mean \pm SD & $0.86 \pm 0.08$ & \\
\hline Postoperative 6 month: Mean \pm SD & $0.82 \pm 0.11$ & \\
\hline Postoperative 12 month: Mean \pm SD & $0.72 \pm 0.15$ & \\
\hline \multicolumn{3}{|c|}{$\mathrm{P} 1=0.003, \mathrm{P} 2<0.0001, \mathrm{P} 3<0.0001, \mathrm{P} 4=0.006, \mathrm{P} 5<0.0001, \mathrm{P} 6<0.0001$} \\
\hline
\end{tabular}

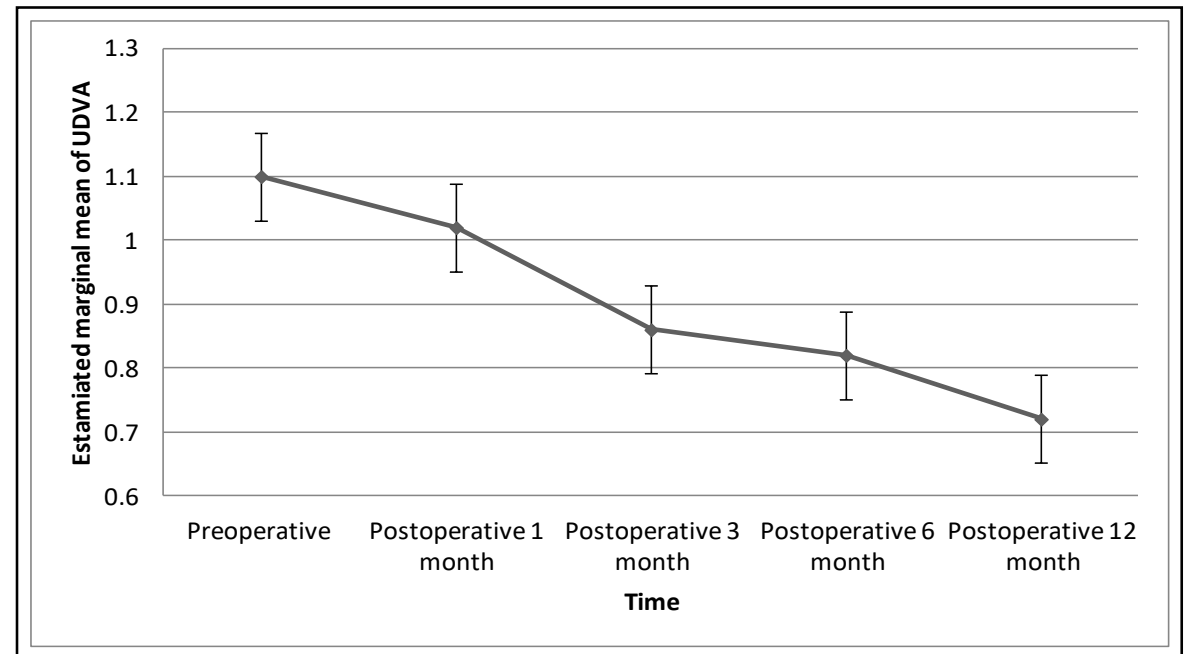

Figure 1: UCVA over time preoperative and postoperative 
Table 2: BCVA over time preoperative and postoperative

\begin{tabular}{|c|c|c|}
\hline Time & BCVA & $P$ value for repeated measures \\
\hline Preop Mean \pm SD & $0.84 \pm 0.09$ & \multirow{5}{*}{$<0.0001$} \\
\hline Postop 1-mth Mean \pm SD & $0.74 \pm 0.16$ & \\
\hline Postop 3-mth Mean \pm SD & $0.68 \pm 0.11$ & \\
\hline Postop 6-mth Mean \pm SD & $0.60 \pm 0.13$ & \\
\hline Postop 12-mth Mean \pm SD & $0.52 \pm 0.14$ & \\
\hline \multicolumn{3}{|c|}{$\mathrm{P} 1<0.0001, \mathrm{P} 2<0.0001, \mathrm{P} 3<0.0001, \mathrm{P} 4<0.0001, \mathrm{P} 5<0.0001, \mathrm{P} 6<0.0001$} \\
\hline
\end{tabular}

p1: compared pre- \& post-operative 3 months, p2: pre-\& post-operative 6 months, p3: pre- \& postoperative 12 months, p4: post-operative 3 months \& post-operative 6 months, p5: post-operative 3 months \& post-operative 12 months, p6: post-operative 6 months \& post-operative 12 months

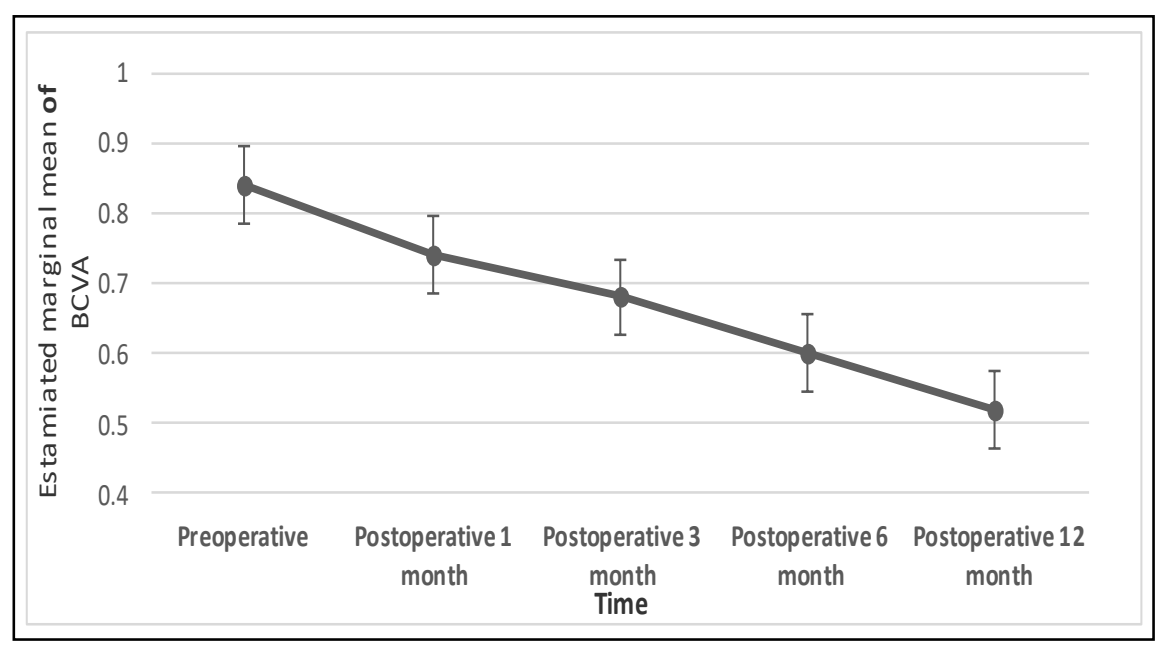

Figure 2: BCVA over time preoperative and postoperative

Table 3: Average K (D) over time preoperative and postoperative

\begin{tabular}{|c|c|c|}
\hline Time & Average K (D) & $P$ value for repeated measures \\
\hline Preop Mean \pm SD & $53.87 \pm 5.42$ & \multirow{5}{*}{0.001} \\
\hline Postop 1 month Mean \pm SD & $52.75 \pm 4.33$ & \\
\hline Postop 3 months Mean \pm SD & $50.26 \pm 2.95$ & \\
\hline Postop 6 months Mean \pm SD & $50.30 \pm 2.58$ & \\
\hline Postop 12 months Mean \pm SD & $51.88 \pm 5.40$ & \\
\hline \multicolumn{3}{|c|}{$\mathrm{P} 1=0.001, \mathrm{P} 2<0.0001, \mathrm{P} 3=0.69, \mathrm{P} 4=1.00, \mathrm{P} 5=0.60, \mathrm{P} 6=0.72$} \\
\hline
\end{tabular}
operative 12 months, p4: post-operative 3 months \& post-operative 6 months, p5: post-operative 3 months \& post-operative 12 months, p6: post-operative 6 months \& post-operative 12 months

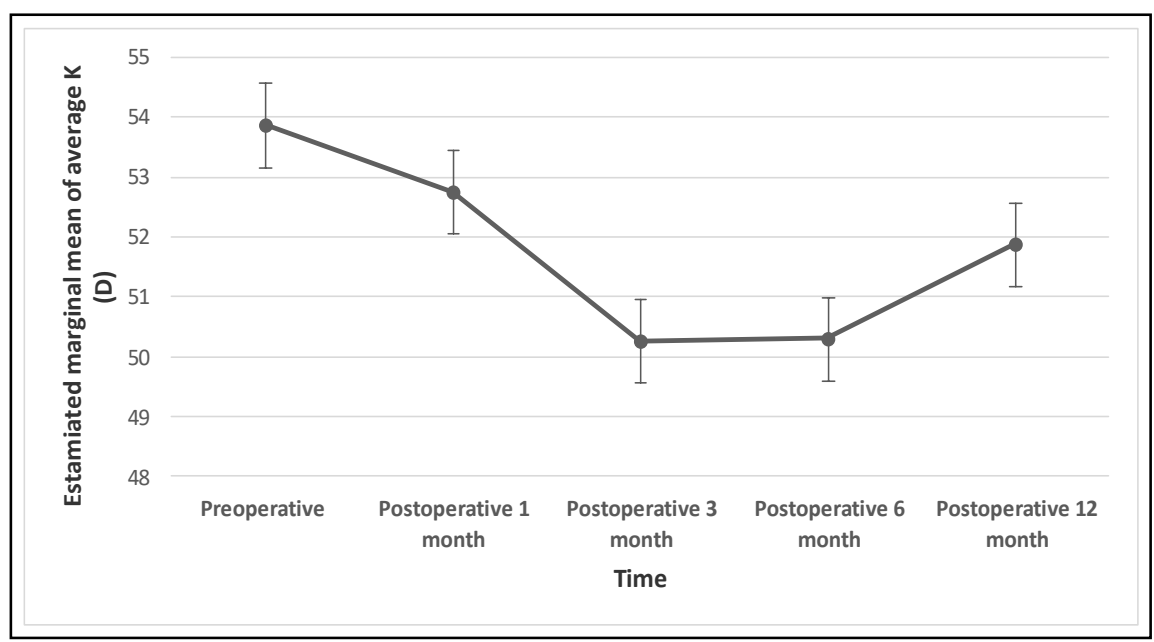

Figure 3: Average K (D) over time preoperative and postoperative 


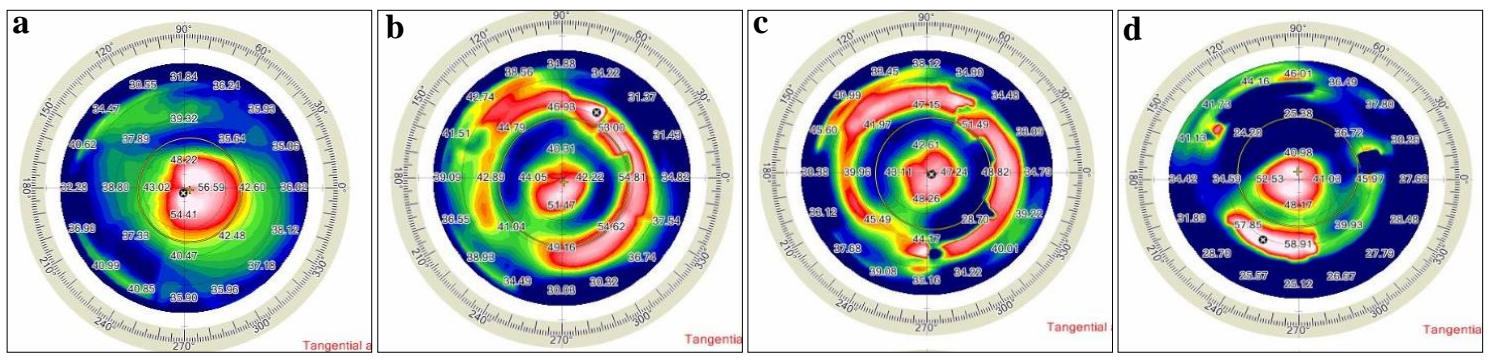

Figure 4: kerometry maps; $\underline{\text { a. }}$ preoperative, $\underline{\mathbf{b}} .1$ month postoperative, $\underline{\mathbf{c}} .3$ month postoperative, $\underline{\mathbf{d}} .1$ year postoperative

\section{Discussion}

Traditionally, the mainstay of management for progressive keratoconus was either penetrating or deep lamellar keratoplasty (PK or DALK, resp.) the success of the procedures, however, was fairly tempered by possible problems and complications both intra operatively or post-operatively, taken together these have been the stimulation for a current searching for less difficult treatment substitutes [7]. These comprise ultra-violet cross-linking (UVCXL) and intra-corneal ring segment (ICRS) both of which were initially can be strained in their indications to eyes with mild to moderate diseases there is today increasing support for their usage in advanced diseases also [8]. The key notion of ICRS implantations is to flatten the cornea and decrease front corneal superficial irregularities, but they actually have no role in halting the progression of keratoconus [9]. Corneal collagen cross linking (CXL) is the real and chief therapy to keratoconus and has the benefit of stopping the progressions of the disorder. The progressions of keratoconus may be described by continues alteration in 2 or more of special factors. These special factors comprised steepening of the backward $\mathrm{K}$ readings, steepening of the frontal $\mathrm{K}$ readings, and thinning of the central pachymetry readings and elevated back superficial raises [10]. CXL PLUS is described as the immediate combinations of CXL and a refractive procedure to flatten the cornea and progress vision as ICRS implantations [11]. Our results were similar to the study done by Saib et al. in France concluded that CXL trailed by ICRS after 4.3 months lead to significant improvements of mean $\mathrm{K}$ readings from 44.8D to 42.5D [12]. Similarly, Saleem et al. investigated 43 eyes of 38 patients with $\mathrm{KC}$, and did femtosecond laser guided Keraring insertions trailed by CXL straightly at one session, and revealed that after 3-yrs K1 significantly reduction from $46.94 \pm 0.26$ to $46.45 \pm$ $0.98-\mathrm{D}$, and $\mathrm{K} 2$ significantly reduced from $54.38 \pm 1.17$ to $47.39 \pm 0.62 \mathrm{D}$ [13]. ICRS has some stabilizing influence upon corneal bio-mechanics, while this impacts continue only about 6-mths, and its role is mostly for correcting the refraction and progress vision [14], which isn't sufficient to stop the development of $\mathrm{KC}$, which needs CXL, that hardens the then weak and flaccid corneal stroma [15]. Managements of progressive corneal ectasia is challenging for any corneal surgeon. Prevention of progression in early stages of the beast way to deal with these disorders [16]. Patient with progressive ectasia could require keratoplasty liable upon the depth of corneal scaring. Penetrating keratoplasty (PK) was the optimal operation for progressive Keratoconus for many years; anterior lameller keratoplasty (ALK) has become the favored therapy. Considering profits counting decreased graft rejections, lesser long-term complication and improved graft existence [17]. However in comparative study of Alnaimy, et al. 50 patients were diagnosed as moderate Keratoconus and divided into 2 equal groups. The $1^{\text {st }}$ group had (DALK) while the $2^{\text {nd }}$ group had femtoassisted keraring implantation. The study concluded that the both treatments were secure and operative 
surgical alternatives in treating cases with moderate Keratoconus and both of them lead to progressive improvement in visual outcome [18]. And also in study of Chen, et al, They reported that epithelium on corneal CXL could improve corneal flattening and higher preoperative keratometry in eyes with advanced Keratoconus [19]. Our conclusion was in agree with Monir's study who reported improvement of visual acuity and corneal parameters counting keratometry and frontal and backward raises in a case of advanced superior Keratoconus treated by accelerated corneal CXL and intra stromal keraring implantation by femtosecond laser [20]. No complications like epithelial faults, white deposits in the segment tunnel, infection, segments migrations to the incision location and corneal vascularization have been detected in our work. The disadvantages in the present work were the short-term following-up, the absence of analysis of corneal deviations and the absence of comparisons with mechanical ICRS (Keraring) implantations.

\section{Conclusion}

In conclusion, CXL appears effective in stabilizing mild to moderate and advanced patients of advanced keratoconus, with further persuaded corneal flattening and elevated corneal regularities. Corneal CXL could possibly diminishes the necessity for corneal transplantations. CXL PLUS showed to be a successful technique to halt progression (principally by CXL) and to correcting the refractive grade of the keratoconic eyes (principally by ICRS). Analysis of the outcome thereafter Keraring ICRS implantations exhibited a significant post-operative corneal flattening with a subsequent rise in UCVA and BCVA. By means of the femto-second laser for tunnel creations made the operation easier and faster with a precise depth of implantations and decreased the danger of surgical and post-operative complications.

\section{References}

1. Barbara, A. Controversies in the management of keratoconus, Springer, Switzerland, 2019.

2. Tsubota, K., Mashima, Y., Murata, H., et al. Corneal epithelium in keratoconus. Cornea. 1995; 14 (1): 77-83.

3. Colin, J., Savary, G. \& Malet, F. Correcting keratoconus with intracorneal rings. J. Cataract Refract Surg. 2000; 26: 1117-1122.

4. Coskuns, even E., Kymionis, G., Tsiklis, N., et al. One-year results of intrastromal corneal ring segment implantation (KeraRing) using femtosecond laser in patients with keratoconus. Am. J. Ophthalmol. 2008. 145: 775-779.

5. Ruckhofer, J., Alzner, E. \& Grabner, G. Confocal microscopy after implantation of intrastromal corneal ring segments. Opthalmology. 2000; 107: 2144-2151

6. Kessler, D. El-Shiaty, A. \& Wachler, B. Evaluation of tear following Intacs for myopia. J. Refract Surg. 2002; 18: 127-129.

7. Parker, J., van Dijk, K. \& Melles, G. Treatment options for advanced keratoconus: A review. Surv. Ophthalmol. 2015; 60 (5): 459-480.

8. Chan, E., Snibson, G. Current status of corneal collagen cross-linking for keratoconus: A review. Clin Exp Optom. 2013; 96: 155e64

9. Kamiya, K., Ishii, R., Shimizu, K., et al. Evaluation of corneal elevation, pachymetry and keratometry in keratoconic eyes with respect to the stage of Amsler-Krumeich classification. Br. J. Ophthalmol. 2014; 98 (4): 459-463.

10. Duncan, J., Belin, M., Borgstrom, M. Assessing progression of keratoconus: Novel tomographic determinants. Eye Vis (Lond). 2016; 3: 6.

11. Randleman JB, Khandelwal SS, Hafezi F. Corneal cross-linking. Surv Ophthalmol. 2015; 60 (6): 509-523 
12. Saib, N., Bonnel, S., Fenolland, J., et al. Intrastromal corneal rings and corneal collagen crosslinking for progressive keratoconus: Comparison of two sequences. Eye. 2015; 29 (2): 294295.

13. Saleem, M., Elzembely, I., Abo Zaid M., et al. Three-year outcomes of crosslinking PLUS (combined cross-linking with femtosecond laser intracorneal ring segments implantation) for management of keratoconus. J. Ophthalmol. 2018; 6907573.

14. Bamdad, S., Sedaghat, M., Yasemi, M., et al. Intracorneal stromal ring can affect the biomechanics of ectatic cornea. J. Ophthalmol. 2020; 4274037.

15. Brown, S., Simmasalam, R., Antonova, N., et al. Progression in keratoconus and the effect of corneal cross-linking on progression. Eye Contact Lens. 2014; 40 (6): 331-338.

16. Maharana, P., Dubey, A., Jhanji, V., et al. Management of advanced corneal ectasias. Br J. Ophthalmol. 2016; 100 (1): 34-40.

17. Patil, M., Mehta, J. Lamellar keratoplasty for advanced keratoconus. Asia Pac J. Ophthalmol (Phila). 2020; 9 (6):580-588.

18. Alnaimy, M., Abou-sharkh, A., Nasr Al-deeb, M., et al. (2017). Evaluation of deep anterior lamellar keratoplasty versus intracorneal ring segments in treating moderate keratoconus. Zagazig Univ. Medical J. 2017; 23 (4): 1-15.

19. Chen, S., Chan, T., Zhang, J., et al. Epithelium-on corneal collagen crosslinking for management of advanced keratoconus. J. Cataract Refract Surg. 2016; 42 (5): 738-749

20. Mounir, A., Mostafa, E. Combined accelerated corneal collagen crosslinking and intrastromal Kerarings implantation for treatment of advanced superior keratoconus. GMS Ophthalmol Cases. 2020; doi:10.3205/oc000137 